\title{
ISO OBSERVATIONS OF AGN AND ULTRALUMINOUS IR GALAXIES
}

\author{
ALAN F. M. MOORWOOD \\ European Southern Observatory \\ Karl-Schwarzschildstr. 2, D-85748 Garching
}

\section{Overview}

All the ISO instruments are contributing to the study of activity in galaxies of essentially all types. Although AGN's as such are pointlike, the beautiful CAM image of CenA shown by Catherine Cesarsky has given us the clearest view so far of its visually obscured nucleus and surrounding spiral disc embedded in an elliptical galaxy. The CAM CVF is also providing spectral images of the PAH features and important diagnostic ionic lines (e.g [NeIl] and [NeIII]) in the circumnuclar regions of $\mathrm{AGN}$ and merging starburst systems (Vigroux et al., 1996). PHT is providing detailed spectral energy distributions over the complete 2.5-240 $\mu \mathrm{m}$ range and PHTS is proving invaluable for assembling a catalogue of low resolution $(\mathrm{R} \simeq 90)$ galaxy spectra covering the $6-12 \mu \mathrm{m}$ PAH features. SWS and LWS are generating higher resolution $(R \simeq 2000-200)$ spectra over the range 2.5$200 \mu \mathrm{m}$ such as that shown in Fig. 1. of the Circinus galaxy which exhibits both AGN and starburst activity and well illustrates the wide range of diagnostic features, many seen for the first time, accessible to ISO studies of galaxies. It shows the overall continuum with a peak around $100 \mu \mathrm{m}$; PAH features and $\mathrm{Si}$ absorption which dominate the spectrum around $10 \mu \mathrm{m} ; \mathrm{H}$ recombination lines; $\mathrm{H}_{2}$ pure rotational emission lines; low ionization potential ionic lines excited by starburst activity and high excitation lines ( $u p$ to $\simeq 300 \mathrm{eV}$ ) excited by the visually obscured AGN.

ISO is still operating and only a fraction of the already collected data have been reduced and analyzed. Nevertheless, important results are already beginning to emerge which can only be briefly summarized in this short contribution.

\section{Some Highlights}

\subsection{WARM DUST}

Several CAM and PHT programmes have already yielded detailed information on the nature of the emission from the warm, AGN heated, dust detected initially by IRAS. Some examples:

- CAM image of the SO Seyfert NGC3998 which separates the extended stellar and AGN heated warm dust component at $\simeq 200 \mathrm{~K}$ (Knapp et al., 1996).

- PHT SED's of CfA Seyferts showing a bi-modal distributions with T's $\simeq 170 / 30 \mathrm{~K}$ (Rodríguez Espinosa, J.M. et al. 1996; Rodríguez Espinosa, J.M. \& Pérez (Tarćá, 1997)

- CAM CVF spectra of the hyperluminous IRAS PO9104+4109 which are consistent with theoretical predictions of the emission from a pc size torus plus a more extended (100pc) component ('Taniguchi et al., 1997).

\subsection{WNRM H:2}

ISO SWS spectra have yiedled the first detections of the pure rotational transitions of molecular hydrogen, including the longest wavelength $0-0 \mathrm{~S}(0)$ line at. $28 \mu \mathrm{m}$. These require the presence of a warm component at $\mathrm{T} \geq 120 \mathrm{~K}$ which, in starburst galaxies, amounts to up to $\simeq 10 \%$ of the total $\mathrm{H}_{2}$ mass deduced from ('O observations (Valentijn et al., 1996; Rigopoulou et al. 1996). In the Seyfert galaxy N(: $106 x$, however, the implied mass of warm $\mathrm{H}_{2}$ is equal to the previously estimated total ( $\mathrm{L}_{411 \%}$ et al. 1997). This is surprising and implies either exceptional conditions or 


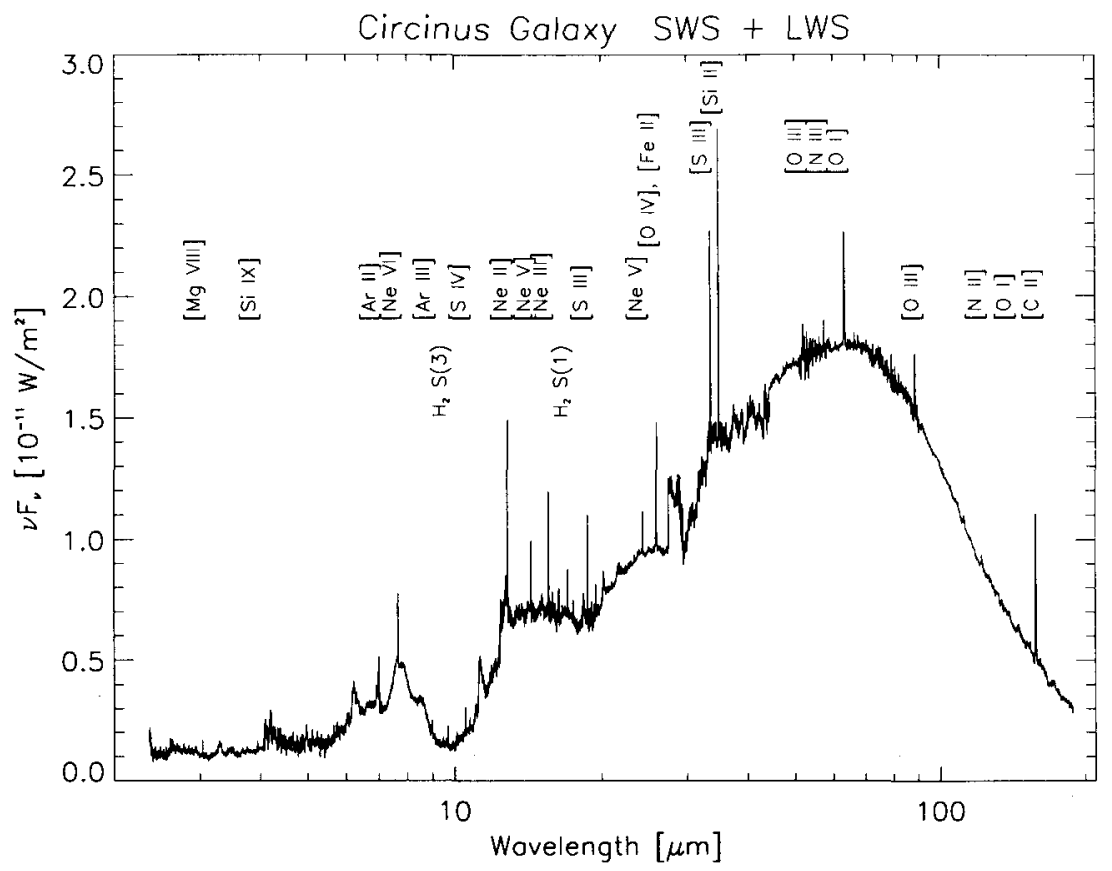

Figure 1. 2.5-200 $\mu \mathrm{m}$ spectrum of the Circinus galaxy.

an underestimation of the cold component e.g related to the much debated value of the $\mathrm{CO} / \mathrm{H}_{2}$ conversion factor.

\subsection{OH MEGAMASERS}

An SWS spectrum of the OH megamaser and Ultraluminous IR galaxy Arp220 showing strong $35 \mu \mathrm{m}$ absorption (Skinner et al. 1997) has finally provided the needed observational confirmation that absorption of infrared continuum photons, most probably from a spatially extended starburst, in this $\mathrm{OH}$ transition is sufficient to pump the radio maser lines. Not only does the number of absorbed photons appear to be sufficient but the velocity width of the absorption is also consistent with that of the radio emission lines as expected. Companion longer wavelength $\mathrm{OH}$ absorptions which may also contribute to the pumping have also subsequently seen with LWS (Fischer et al. 1997).

\subsection{EUV CONTINUUM OF AGN}

Circinus provides the closest $(\simeq 4 \mathrm{Mpc})$ example of a galaxy exhibiting both starburst activity and a prominent ionization cone originating in an embedded Seyfert type 2 nucleus. The SWS part of its spectrum shown in Fig. 1 has provided the first density estimate for the high excitation gas from the $[\mathrm{NeV}](14.3 / 24.3 \mu \mathrm{m})$ line ratio and contains a wide range of emission lines from species with ionization energies up to $\simeq 300 \mathrm{eV}$ which can be used to probe its obscured EUV continuum. Assuming radiation bounded clouds, its infrared emission line spectrum is accurately reproduced by a photoionization model in which the ionizing source spectrum is represented by a broken power law $\left(\nu^{-0.5}\right)$ with a strong $(\mathrm{x} 10)$ break around $70 \mathrm{eV}$ similar to that inferred in Seyfert 1's and quasars (Moorwood et al., 1996). This spectrum is also consistent with $10 \mathrm{keV}$ x-ray observations and its integrated luminosity is sufficient to power most or all of the observed far infrared luminosity. 
Spectra of other AGN's of various types (NGC4151 S1, NGC1068 S2, CenA RG, NGC7582 NLX) imply ionizing spectra which are similar although somewhat softer than that of Circinus in the EUV range (Moorwood, 1997) and more detailed modelling of these galaxies is in progress.

\subsection{NATURE OF ULTRALUMINOUS IR GALAXIES}

A prime goal of ISO is to resolve the controversial question as to the relative importance of AGN and starbursts in powering the Ultraluminous IR galaxies discovered by IRAS. High quality observations of the prototype, Arp220, have already been obtained with all the ISO instruments. It exhibits a bi-modal continuum SED with a somewhat cooler warm component than in Seyferts (Klaas et al., 1997); PAH feature emission (Charmandaris et al., 1997; Genzel et al., 1997); low (e.g [NeII], [SIII]) but not high ([OIV]) excitation lines in the mid-IR (Sturm et al., 1996) and, somewhat surprisingly, no or extremely weak emission lines but strong molecular absorption features in the far infrared (Fischer et al., 1997). Of particular interest is the faintness of the [CII] $(158 \mu \mathrm{m})$ line, emitted by photodissociation regions, which has been found to be similarly weak in other Ultraluminous galaxies for reasons which are still not well understood but could include self absorption or different UV field/density ratios than those typical of lower luminosity starbursts (Luhman et al., 1997). The most extensive study so far has used both the SWS and PHTS to observe a sample of 15 ULIRG's plus 12 starburst and 16 AGN template galaxies (Lutz et al., 1996; Genzel et al., 1997). An important result is that starburst galaxies and AGN's are well separated in a plot of [OIV]/[NeII] versus $7.7 \mu \mathrm{m}$ PAH EW. AGN's exhibit $[\mathrm{OIV}] \simeq[\mathrm{NeII}]$ and no or weak $\mathrm{PAH}$ emission while starbursts show no or weak [OIV] but strong PAH emission. The ULIRG's represent an intermediate case but mostly lie closer to the starbursts with only two showing high excitation ([OIV] or [NeV]) lines. They are characterized by higher $\mathrm{L}_{I R} / \mathrm{L}_{L y c}$ ratios than lower luminosity starbursts but only $\simeq \mathrm{x} 3$ which is lower than previous estimates following correction for the higher extinction values derived from the mid-IR compared with earlier near-IR spectra. Although some or all could contain AGN, therefore, it is presently concluded that the luminosity in most of the sample observed so far is dominated by a starburst which is more heavily dust embedded and may be, or at least appear, somewhat older than the well known lower luminosity ones.

\section{Acknowledgements}

I am grateful to D. Lutz and E. Sturm for preparing Fig. 1. and to V. Charmandaris, J. Fischer, R. Genzel, U. Klaas, and J. Rodriguez Espinosa for permission to present their unpublished results.

\section{References}

Charmandaris, V., Mirabel, F., Tran, et al. 1997, to appear in Mamon, Trinh Xuân Thuân \& Trân Thanh Vân (eds.) Extragalactic Astronomy in the Infrared, Editions Frontiéres.

Fischer, J.,Satyapal, S., Luhman, M.L., et al. 1997, to appear in Mamon, Trinh Xuân Thuân \& Trân Thanh Vân (eds.) Extragalactic Astronomy in the Infrared, Editions Frontiéres.

Genzel, R., Lutz, D., Sturm, E., et al. 1997, ApJ (submitted)

Klaas, U., Haas, M., Heinrichsen, 1. \& Schulz, B. 1997, A\&A (in press)

Knapp G.R., Rupen, M.P., Fich, M., Harper, D.A. \& Wynn-Williams, C.G. 1996, A\&A, 315, L75

Luhman, M.L., Fischer, J., Satyapal, S., et al. 1997, to appear in Mamon, Trinh Xuân Thuân \& Trân Thanh Vân (eds.) Extragalactic Astronomy in the Infrared, Editions Frontiéres.

Lutz, D, Genzel, R., Sternberg, A. et al. 1996, A\&A, 315, L137

Lutz, D., Sturm, E., Genzel, R., et al 1997, Proceedings of the NGC1068 Ringberg Workshop (eds J.F. Gallimore \& L.J. Tacconi), to appear in Ap\&SS.

Moorwood, A.F.M., Lutz, D., Oliva, E., et al. 1996, A\&A, 315, L109

Moorwood, A.F.M. 1997, to appear in Mamon, Trinh Xuân Thuân \& Trân Thanh Vân (eds.) Extragalactic Astronomy in the lnfrared, Editions Frontiéres.

Taniguchi, Y., Sato, Y., Kawara, K., Murayama, T. \& Mouri. H. 1997, A\&A, 318, L1

Rigopoulou, D., Lutz, D., Genzel, R., et al. 1996, A\&A, 315, L125

Rodriguez Espinosa, J.M. \& Pérez Garcia, A.M., Lemke, D., et al. 1996, A\&A, 315, L129

Rodriguez Espinosa, J.M. \& Pérez García, A.M. 1997, A\&A (submitted)

Skinmer, C.J., Smith, H.A., Sturm, E. et al. 1997, Nature, 386, 472

Sturm, E., Lutz, D., Genzel, R, et al. 1996, A\&A, 315, L133

Valentijn, E.A., van der Werf, P.P., de Graauw, Th. \& de Jong, T. 1996, A\&A, 315. L145

Vigroux, L., Mirabel, F., Altieri, B. et al. 1996, A\&A, 315, L93 